\title{
Limnology of Two Contrasting Hydroelectric Reservoirs (Storage and Run-of-River) in Southeast Brazil
}

\author{
Marcos Gomes Nogueira, \\ Gilmar Perbiche-Neves and Danilo A. O. Naliato \\ Instituto de Biociências, \\ UNESP - Universidade Estadual Paulista Rubião Junior s/n, São Paulo, \\ Brazil
}

\section{Introduction}

The present chapter is a contribution to the discussion about the limnological differences between storage and run-of-river reservoirs. We also intend to contribute to the improvement of the use of this operational concept in an ecological perspective.

Artificial reservoirs created by the construction of dams along fluvial systems tend to exhibit intermediate limnological characteristics, as compared to rivers and lakes. The tendency of a reservoir to be more similar either to a lotic or to a lentic environment is basically related to the water retention time. As shorter is the retention time the similarity with a river is higher. Conversely, the structure and functioning of a reservoir will be comparable to a lake in case of high water retention time. In both cases it is generally observed conspicuous spatial gradients, represented by riverine, intermediate and lentic zones, in the main water body of the reservoir as well as in the lateral arms, in case of dendritic systems (Thornton, 1990; Tundisi, 1990).

Vertically, the reservoirs also have a complex structure, influenced by seasonal variation in the processes of formation and breakdown of thermal stratification and depth of the thermocline (Han et al., 2000), as well as by the plant operation, especially the vertical positioning of the water intake to the turbines and total outflow (Naliato et al., 2009).

The construction of large reservoirs for hydroelectric production in Brazil was intensive after the 1960's, especially in the southeast and south hydrographic basins. Presently, it is estimated that only $40 \%$ of the main rivers of the Paraná basin, second largest one in the continent after the Amazonian, are still free of damming (Agostinho et al., 2007). The reservoirs of this basin can be classified, in terms of the operational design and engineering concept, as accumulation (storage) or run-of-river systems (Kelman et al., 1999). The accumulation systems are larger in size and volume, with high water retention time The distinctiveness in terms of physical dimensions and functioning between accumulation and run-of-river systems affects the physical and chemical limnology of these environments as 
well as the structure and functioning of the aquatic communities (Nogueira et al., 2008; Perbiche-Neves \& Nogueira, 2010; Tundisi \& Matsumura-Tundisi, 2003). Additionally, the erosive processes in the river banks downstream the dam can be intensified as a result of the different regimes of water discharges (Stevaux et al., 2009).

Studies carried out in Brazilian reservoirs indicate that the main factors influencing in the limnological conditions - seasonal and spatial patterns, are the latitude, the lake and watershed morphometry and the water retention time (e.g Naliato et al., 2009; Henry et al., 1995; Soares et al., 2009).

In the Paranapanema River, where the studied reservoirs are located, continuous limnological investigations have been carried out in the last two decades. Among the relevant results it can be mentioned the ones published by Henry (1992), Henry \& Gouveia (1993), Henry \& Maricatto (1996), Nogueira et al. (1999) and Nogueira et al. (2006).

Eleven reservoirs were constructed in the Paranapanema River (between 1950's and 1990's), in approximately $500 \mathrm{~km}$ of river stretch, in order to supply electric power (ca. 1,500 MW) for an integrated national system of distribution. Three of these reservoirs are accumulation systems, dendritic in shape, larger than $400 \mathrm{~km}^{2}$ in area and with retention time longer than 130 days. The others are run-of-river systems with retention time not longer than to 22 days (Nogueira et al., 2006).

The main objective of the present study was to verify the distinctiveness between two kind of reservoirs, storage (Chavantes) and run-of-river (Salto Grande), despite of their geographical proximity. It was also investigated the hypothesis that the limnological conditions in the run-of-river reservoir, due to its small size and low retention time, are similar to the ones observed in the dam (lacustrine) zone of the accumulation reservoir, upstream located.

\section{Material and methods}

As previously mentioned, the studied reservoirs are located in the Paranapanema River, in the border of the States of São Paulo and Paraná (Brazil). Chavantes is an accumulation (storage) reservoir and Salto Grande is a run-of-river reservoir. The distance between dams is approximately $50 \mathrm{~km}$ and the distance between the most upstream sampling point and the most downstream one is about $105 \mathrm{~km}$. There is no large cities in the region, only towns and one middle size municipality (Ourinhos municipality, 104,000 inhabitants) whose urban effluents are discharged in Salto Grande Reservoir through the tributary Pardo River. The land use is less intensive in Chavantes watershed, due to the relief limitation (acclivity). But the agriculture practices are intensive around Salto Grande Reservoir. The superficial area of Chavantes is $400 \mathrm{~km}^{2}$, the theoretical water retention time is 418 days (annual mean value), the maximum depth is $90 \mathrm{~m}$ and it is considered as an oligomesotrophic system. Salto Grande is $12 \mathrm{~km}^{2}$ in size, retention time of 1.5 days (annual mean value), maximum depth of $15 \mathrm{~m}$ and it is considered as a eutrophic system after the Pardo River entrance. Additional information on the reservoirs can be found in Pagioro et al. (2005a), Nogueira et al. (2006), Nogueira et al. (2008), Perbiche-Neves \& Nogueira (2010) and Perbiche-Neves et al. (2011). Between Chavantes and Salto Grande there is another small reservoir (Ourinhos Dam) which started to operate for electric generation, just after the beginning of the study.

The theoretical water retention time (WRT) for both reservoirs was calculated through the relation between volume and outflow. The volumes were considered as constant (normal 
operational cota) and for the outflow it was used the monthly mean value, calculated from continuous data registration (hourly measurements).

Samplings were performed quarterly, between October/2005 and July/2007 in Chavantes, and between November/2005 and August/2007 in Salto Grande. Six sampling stations were determined in Chavantes and 5 in Salto Grande. Figure 1 shows the sampling sites geographic position, maximum depth and trophic state classification (Carlson Index) (after Mercante \& Tucci-Moura, 1999). The distribution of the sampling points was planned in order to include the main compartments in terms of the system hydrodynamics. In the lateral rivers (points VR, IR, PRM e NB), the water samples were obtained only at the subsurface (about $20 \mathrm{~cm}$ depth), due to the predominant small depth and turbulent condition. For the other sampling points samples were obtained in 4 different depths (Van Dorn bottle). The water samples were used for determination of total nitrogen (Mackereth et al., 1978) and total phosphorus (Strickland \& Parsons, 1960), after digestion procedure (Valderrama, 1981), a chlorophyll (Talling \& Driver, 1963), suspended matter (total, mineral and organic) (Cole, 1979) and turbidity (MS Tecnopon). In each sampling point a vertical profile of temperature, dissolved oxygen, $\mathrm{pH}$, conductivity and redox potential was measured using a Horiba U-22 probe. The transparency (Secchi disk) and depth (Speed Tech sonar) were also measured.

Except for the tributary rivers (one sampling depth), it was calculated the data mean values and standard deviation. For statistical analyses the values were log transformed, except for pH. Pearson correlation analysis, Statistic 7.0 (Statsoft, 2006), between WRT and outflow discharges, was performed. The ANOVA test, Statistic 7.0 (Statsoft, 2006), was used to verify the spatial and temporal variations of the limnological variables.

A PCA analysis was used for ordination (Kindt \& Coe, 2005) of the sampling points and sampling periods ("R Development Core Team", 2006). This analysis calculates the scores (principal components) which concentrates the variables information and reduce their dimensions.

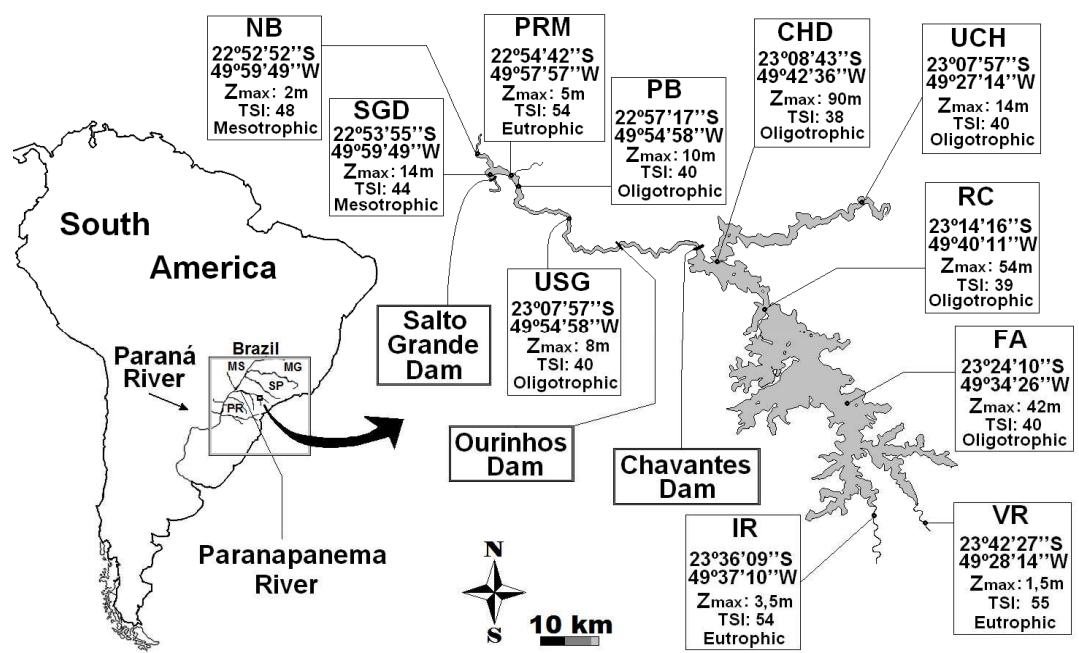

Fig. 1. Study area and the geographical positioning of the sampling stations location with information of the maximum depth (Zmax) and the trophic state index (TSI). 


\section{Results}

During the study period the seasonal regime of rains was typical and similar for both watersheds (Chavantes and Salto Grande Reservoirs), with higher precipitation in summer (December - February) and a dry period in autumn/winter (April - September) (Figure 2). Large scale climatic phenomenon, such as "El Niño" or "La Niña", did not occur during the studied years.

The theoretical water retention in Chavantes during the study period was high (mean of 374 days) and varied between 200 days (winter - August 2006) and 500 days (autumn/winter May to July 2007). The amplitude of variation of the water level was also high (4.5 m). It was associated with the rain seasonality and exhibited a remarkable decrease in autumn and winter 2006. A sudden elevation of the water level was seen in the beginning of summer (December/06) (Figure 3). Seasonal variation in the water level was not observed in Salto Grande. However, there was a high frequency (daily) of low amplitude (ca. $15-20 \mathrm{~cm}$ ) variation in this run-of-river reservoir (Figure 3), where the water retention time was only 1.39 days (mean value) during the study period.
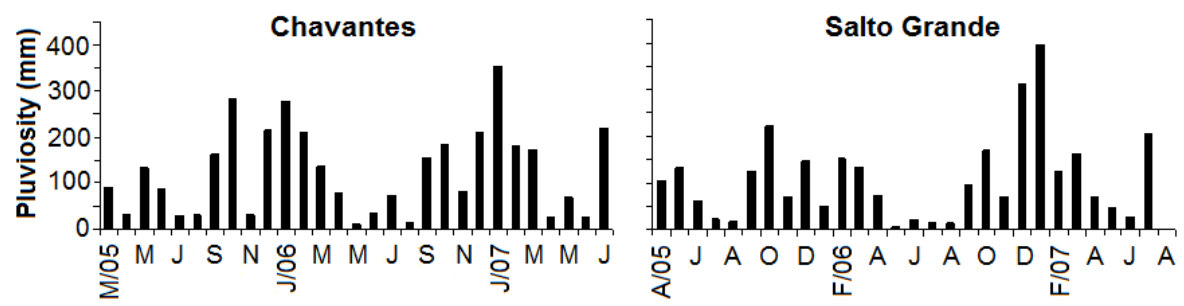

Fig. 2. Monthly rain variation in the watersheds of Chavantes and Salto Grande Reservoirs during the study period.
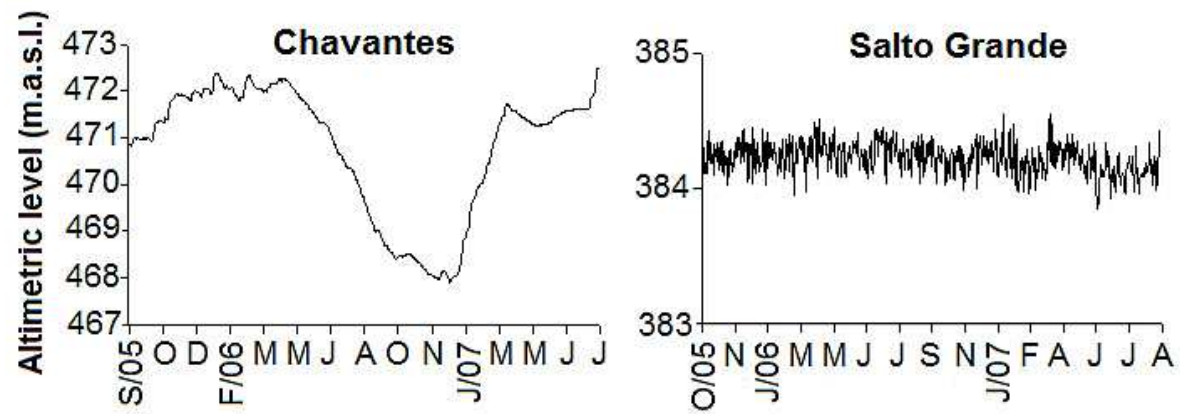

Fig. 3. Daily variation of the water level in Chavantes and Salto Grande Reservoirs during the study period.

The outflow rate for both reservoirs was inversely and significantly correlated with the water retention time, as expected (Figure 4). The water retention time was higher in the second study year $(2006 / 2007)$ compared to the first $(2005 / 2006)$. This inter year variation is probably determined by the needs of regulation (volume control) in the downstream reservoirs of the cascade. Despite the differences in magnitude there was a similar tendency of variation for both reservoirs, what demonstrates the strong influence of the upstream system. 

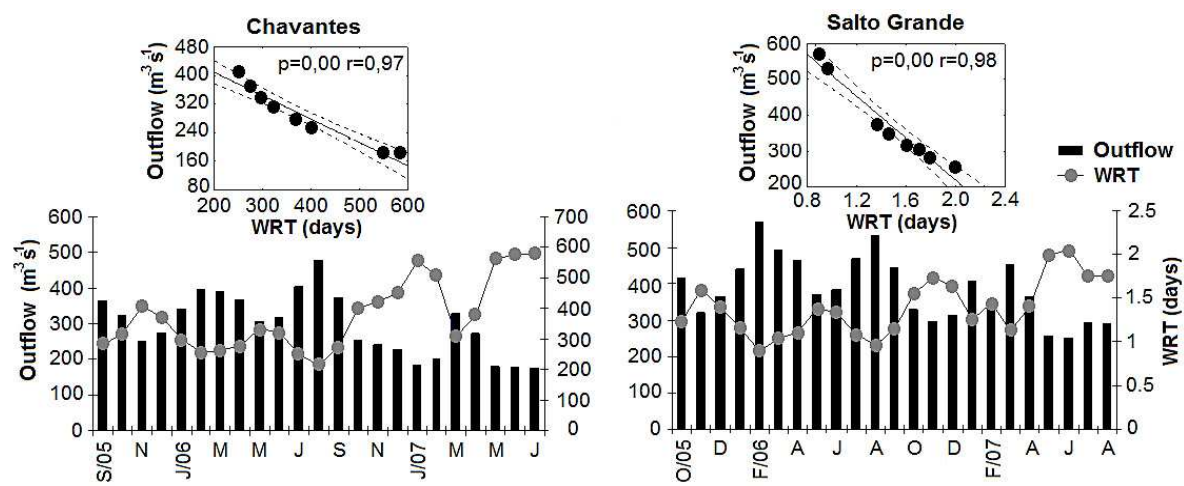

Fig. 4. Monthly variation of outflow and water retention time in Chavantes and Salto Grande Reservoirs during the study period.

The intra reservoir variation of the water transparency was distinct when considered both reservoirs (Figure 5). In the accumulation system there was a clear longitudinal increase towards the dam. Conversely, in the run-of-river reservoir the transparency decreased in the dam zone, due to the entrance of turbid waters from the tributaries (Pardo and Novo). Higher transparency in the Salto Grande upstream sampling station is directly correlated to the lacustrine water releases from Chavantes.

Seasonally the transparency increased in the autumn/winter for both reservoirs (e.g. July in Chavantes) due to the decrease in precipitation and, as a consequence, in the entrance of suspended solids from the adjacent soils and tributaries.
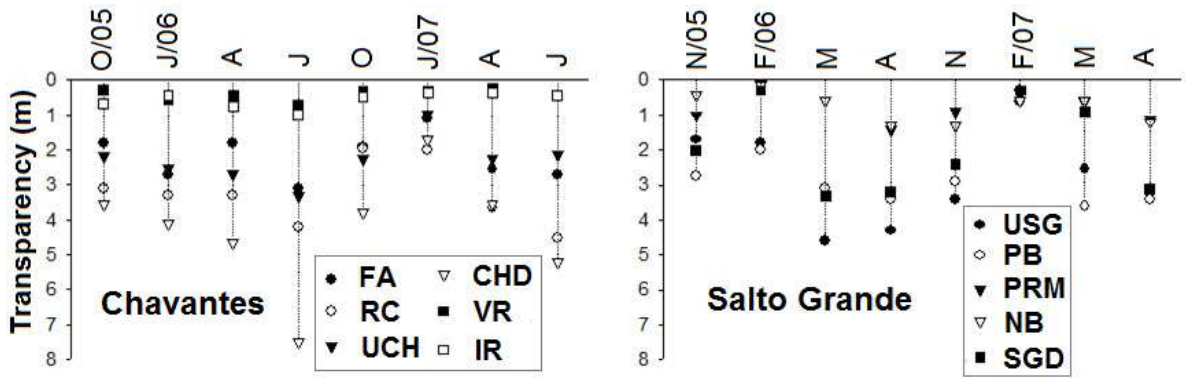

Fig. 5. Water transparency variation in Chavantes and Salto Grande Reservoirs during the study period. For abbreviations see Figure 1.

The vertical profiles data showed the existence of well-structured thermal stratification in deeper sampling stations of Chavantes Reservoir during summer. At the sampling stations $\mathrm{CHD}, \mathrm{RC}$ and FA differences up to $7^{\circ} \mathrm{C}$ were observed along the water column; with remarkable decreases not only of the temperature, but also of $\mathrm{pH}$ and dissolved oxygen bellow $30 \mathrm{~m}$. Homogeneous physical and chemical conditions prevailed in all sampling stations during winter.

In Salto Grande there was no thermal stratification. Only a weak tendency of stratification, probably a daily phenomenon, was seen in the dam zone (SGD) and, episodically, at NB. 
The contrasting conditions in terms of the vertical structure of the water column is shown in Figure 6, which exhibits detailed profiles of temperature and oxygen in the dam zones of Chavantes and Salto Grande during summer and winter.

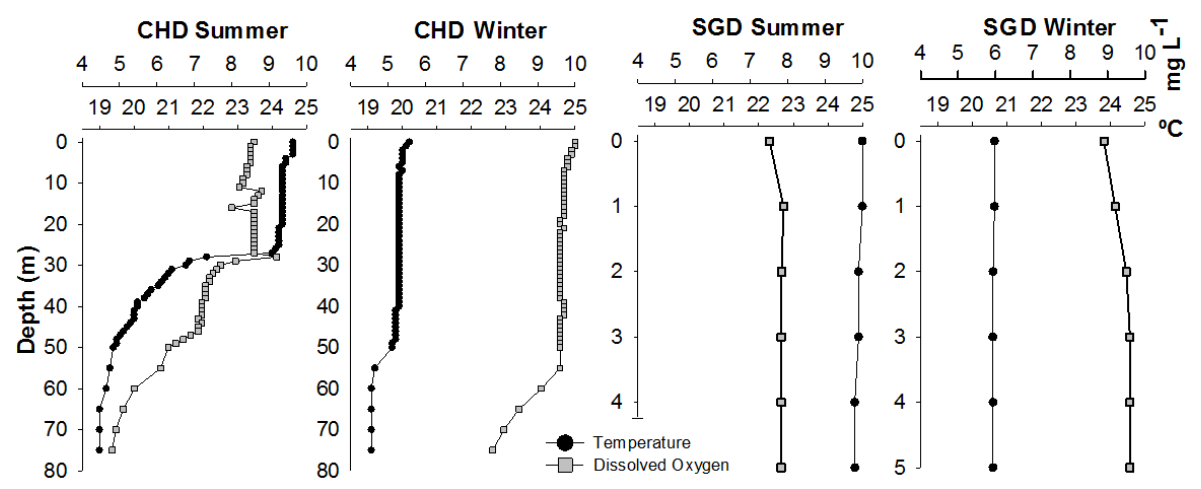

Fig. 6. Vertical profiles of temperature and dissolved oxygen in the dam zones of Chavantes (CHD) and Salto Grande (SGD) Reservoirs.

The mean values and standard deviation of the variables temperature, $\mathrm{pH}$, dissolved oxygen, electric conductivity, turbidity, suspended solids, total phosphorus, total nitrogen an chlorophyll are shown in Tables 1 and 2 for Chavantes and Table 3 for Salto Grande.

In case of Chavantes, the influence of stratified conditions, previously mentioned, is indicated by high standard deviations for temperature, dissolved oxygen and $\mathrm{pH}$.

Clear spatial and temporal differences were observed. Comparatively higher values of temperature were measured in lotic environments during summer and lower in the winter.

The $\mathrm{pH}$ variation was higher in the lentic compartments of the accumulation reservoir, due to the influence of stratification and higher depths. Acid values were detected in the deeper layers. Similar pattern occurred for the oxygen.

The distribution of the conductivity values was not influenced by stratification processes. For Chavantes Reservoir higher values, about $100 \mu \mathrm{S} \mathrm{cm}^{-1}$, were associated to the tributary Itararé (IR) and lower values, about $30 \mu \mathrm{S} \mathrm{cm}-1$, to the tributary Verde (VR), indicating distinct geological composition of these sub basins. For Salto Grande Reservoir the variability of conductivity was also determined by the tributaries entrance. It was higher in Pardo River Mouth (PRM), about $120 \mu \mathrm{S} \mathrm{cm}^{-1}$, and lower in Novo River Bay (NB), about 45 $\mu \mathrm{S} \mathrm{cm} \mathrm{cm}^{-1}$.

Higher mean values of turbidity were detected in the tributaries mouths for both reservoirs. The highest value was verified in the Verde River (VR), 810 NTU in April/2007. The same tendency of variation was seen for suspended solids. A diminution of turbidity and suspended solids occurred towards the dam of Chavantes (CHD). But this tendency was not observed for Salto Grande due to the Pardo River entrance, as already mentioned in the description of the transparency variation. Both variables were also influenced by stratification, with increments in deeper layers of Chavantes sampling stations during warmer periods. 
Limnology of Two Contrasting

Hydroelectric Reservoirs (Storage and Run-of-River) in Southeast Brazil

\begin{tabular}{|c|c|c|c|c|c|c|c|}
\hline & & $\begin{array}{c}\text { Temp. }\left({ }^{\circ} \mathrm{C}\right) \\
\text { M DD.P }\end{array}$ & $\begin{array}{c}\mathrm{pH} \\
\mathrm{M} \pm \mathrm{D} . \mathrm{P}\end{array}$ & $\begin{array}{c}\text { O.D. }\left(\mathrm{mg} \mathrm{L}^{-1}\right) \\
\text { M DD.P }\end{array}$ & $\begin{array}{c}\mathrm{TP}(\mu \mathrm{g} \mathrm{L}-1) \\
\mathrm{M} \pm \mathrm{D} . \mathrm{P}\end{array}$ & $\begin{array}{c}\text { TN }(\mu \mathrm{g} \mathrm{L}-1) \\
\text { M } \pm \text { D.P }\end{array}$ & $\begin{array}{c}\text { Chlor. ( } \mu \text { g L-1) } \\
\text { M D.P }\end{array}$ \\
\hline \multirow{8}{*}{$\mathrm{UCH}$} & Oct/05 & $23.23 \pm 0.20$ & $8.84 \pm 0.39$ & $7.98 \pm 0.08$ & $22.13 \pm 1.70$ & $403.08 \pm 59.30$ & $1.87 \pm 0.64$ \\
\hline & Jan/06 & $27.60 \pm 0.69$ & $6.73 \pm 1.02$ & $6.63 \pm 0.62$ & $38.97 \pm 4.28$ & $409.26 \pm 16.75$ & $1.87 \pm 0.72$ \\
\hline & Apr/06 & $23.81 \pm 0.04$ & $8.30 \pm 0.00$ & $6.64 \pm 0.17$ & $11.58 \pm 0.58$ & $122.90 \pm 20.70$ & $1.16 \pm 0.09$ \\
\hline & Jul/06 & $19.04 \pm 0.17$ & $10.27 \pm 0.16$ & $6.71 \pm 0.11$ & $11.71 \pm 2.77$ & $393.79 \pm 45.64$ & $1.12 \pm 0.20$ \\
\hline & Oct/06 & $22.58 \pm 0.26$ & $6.72 \pm 0.04$ & $9.95 \pm 0.12$ & $16.82 \pm 0.94$ & $352.10 \pm 6.61$ & $1.66 \pm 0.59$ \\
\hline & Jan/07 & $25.90 \pm 0.02$ & $6.15 \pm 0.02$ & $8.36 \pm 0.06$ & $12.98 \pm 1.98$ & $289.15 \pm 76.47$ & $1.15 \pm 0.33$ \\
\hline & Apr $/ 07$ & $25.78 \pm 0.09$ & $4.82 \pm 0.01$ & $6.96 \pm 0.05$ & $9.15 \pm 0.99$ & $266.98 \pm 34.78$ & $1.15 \pm 0.24$ \\
\hline & Jul/07 & $19.24 \pm 0.12$ & $6.30 \pm 0.02$ & $9.61 \pm 0.07$ & $6.11 \pm 0.41$ & $276.87 \pm 30.68$ & $1.50 \pm 0.56$ \\
\hline \multirow[t]{8}{*}{$\mathrm{FA}^{*}$} & Oct/05 & $20.98 \pm 1.12$ & $9.28 \pm 0.32$ & $7.87 \pm 0.46$ & $40.02 \pm 0.54$ & $443.03 \pm 93.39$ & $2.47 \pm 1.24$ \\
\hline & Jan/06 & $24.43 \pm 2.33$ & $5.31 \pm 1.97$ & $6.44 \pm 0.80$ & $36.95 \pm 3.30$ & $320.85 \pm 11.27$ & $1.72 \pm 1.57$ \\
\hline & Apr/06 & $22.88 \pm 1.00$ & $7.39 \pm 0.97$ & $6.80 \pm 0.30$ & $11.38 \pm 1.58$ & $129.09 \pm 69.96$ & $1.03 \pm 0.08$ \\
\hline & Jul/06 & $19.49 \pm 0.08$ & $9.49 \pm 0.08$ & $6.93 \pm 0.36$ & $12.57 \pm 1.79$ & $466.48 \pm 54.48$ & $1.15 \pm 0.32$ \\
\hline & Oct/06 & $20.53 \pm 1.36$ & $10.32 \pm 0.32$ & $6.82 \pm 0.19$ & $15.74 \pm 7.88$ & $422.08 \pm 30.83$ & $1.89 \pm 0.68$ \\
\hline & Jan/07 & $23.62 \pm 1.92$ & $6.87 \pm 1.27$ & $5.82 \pm 0.28$ & $7.99 \pm 0.99$ & $338.70 \pm 29.26$ & $1.35 \pm 0.33$ \\
\hline & Apr $/ 07$ & $24.16 \pm 2.02$ & $4.71 \pm 2.53$ & $4.05 \pm 0.65$ & $8.18 \pm 1.68$ & $227.70 \pm 13.50$ & $1.35 \pm 0.80$ \\
\hline & Jul/07 & $19.92 \pm 0.02$ & $8.71 \pm 0.04$ & $6.07 \pm 0.04$ & $4.88 \pm 0.67$ & $320.33 \pm 64.43$ & $1.09 \pm 0.57$ \\
\hline \multirow{8}{*}{$\mathrm{RC}^{*}$} & Oct/05 & $21.85 \pm 1.79$ & $8.88 \pm 0.63$ & $7.61 \pm 0.69$ & $28.24 \pm 2.86$ & $412.61 \pm 89.31$ & $2.55 \pm 0.62$ \\
\hline & Jan/06 & $26.46 \pm 2.46$ & $5.76 \pm 1.72$ & $7.00 \pm 0.82$ & $31.55 \pm 6.60$ & $244.30 \pm 69.06$ & $3.00 \pm 0.75$ \\
\hline & Apr $/ 06$ & $23.39 \pm 1.56$ & $7.40 \pm 1.07$ & $6.99 \pm 0.25$ & $9.66 \pm 1.49$ & $70.06 \pm 51.33$ & $1.50 \pm 0.57$ \\
\hline & Jul/06 & $19.93 \pm 0.29$ & $9.22 \pm 0.22$ & $6.67 \pm 0.19$ & $10.52 \pm 2.86$ & $382.19 \pm 62.09$ & $1.19 \pm 0.51$ \\
\hline & Oct/06 & $21.63 \pm 1.84$ & $11.28 \pm 0.50$ & $6.98 \pm 0.34$ & $15.21 \pm 1.35$ & $366.63 \pm 12.06$ & $1.79 \pm 0.39$ \\
\hline & Jan/07 & $22.82 \pm 3.25$ & $6.66 \pm 1.41$ & $6.30 \pm 0.57$ & $10.18 \pm 2.31$ & $305.93 \pm 51.29$ & $1.89 \pm 0.26$ \\
\hline & Apr $/ 07$ & $23.97 \pm 2.64$ & $5.00 \pm 1.97$ & $5.88 \pm 0.50$ & $6.78 \pm 1.72$ & $249.71 \pm 57.26$ & $1.89 \pm 0.80$ \\
\hline & Jul/07 & $20.15 \pm 0.11$ & $7.30 \pm 0.43$ & $5.98 \pm 0.05$ & $6.82 \pm 1.05$ & $234.06 \pm 21.36$ & $1.08 \pm 0.85$ \\
\hline \multirow[t]{8}{*}{$\mathrm{CHD}^{*}$} & Oct/05 & $21.85 \pm 1.42$ & $8.97 \pm 0.47$ & $7.75 \pm 0.30$ & $36.42 \pm 3.90$ & $457.71 \pm 140.87$ & $2.89 \pm 0.92$ \\
\hline & Jan/06 & $24.80 \pm 2.65$ & $5.68 \pm 1.74$ & $6.43 \pm 0.67$ & $36.06 \pm 2.31$ & $317.76 \pm 65.35$ & $3.46 \pm 1.34$ \\
\hline & Apr $/ 06$ & $22.53 \pm 2.04$ & $7.72 \pm 1.05$ & $7.22 \pm 0.46$ & $8.89 \pm 1.03$ & $58.20 \pm 7.38$ & $1.67 \pm 0.35$ \\
\hline & Jul/06 & $19.89 \pm 0.08$ & $9.58 \pm 0.37$ & $6.79 \pm 0.37$ & $14.00 \pm 6.05$ & $321.14 \pm 28.20$ & $0.99 \pm 0.42$ \\
\hline & Oct/06 & $20.48 \pm 1.60$ & $10.65 \pm 0.37$ & $6.37 \pm 0.29$ & $13.29 \pm 2.45$ & $357.43 \pm 16.76$ & $1.13 \pm 0.46$ \\
\hline & Jan/07 & $22.57 \pm 3.06$ & $6.73 \pm 1.02$ & $6.33 \pm 0.28$ & $11.89 \pm 1.55$ & $300.22 \pm 67.52$ & $1.18 \pm 0.22$ \\
\hline & Apr/07 & $22.85 \pm 2.94$ & $4.91 \pm 1.81$ & $5.86 \pm 0.40$ & $9.27 \pm 1.44$ & $218.33 \pm 55.66$ & $1.18 \pm 0.97$ \\
\hline & Jul/07 & $21.85 \pm 1.42$ & $5.91 \pm 0.01$ & $8.30 \pm 0.00$ & $6.40 \pm 0.40$ & $168.33 \pm 22.46$ & $1.67 \pm 0.42$ \\
\hline \multirow[t]{8}{*}{$\mathrm{VR}^{* *}$} & Oct/05 & 24.80 & 7.26 & 8.00 & 35.42 & 533.75 & 3.37 \\
\hline & Jan/06 & 27.80 & 7.30 & 5.27 & 51.88 & 468.02 & 9.83 \\
\hline & Apr/06 & 21.40 & 6.90 & 8.90 & 23.93 & 120.83 & 2.64 \\
\hline & Jul/06 & 16.70 & 6.47 & 11.30 & 22.21 & 523.70 & 1.24 \\
\hline & Oct/06 & 25.25 & 6.43 & 8.90 & 50.85 & 633.50 & 1.30 \\
\hline & Jan/07 & 26.86 & 6.63 & 6.80 & 27.99 & 642.95 & 6.20 \\
\hline & Apr/07 & 23.55 & 5.35 & 6.40 & 69.98 & 679.80 & 5.16 \\
\hline & Jul/07 & 16.22 & 5.65 & 10.30 & 34.86 & 574.80 & 2.69 \\
\hline \multirow[t]{8}{*}{$\mathrm{IR}^{* *}$} & Oct/05 & 24.00 & 7.40 & 8.16 & 42.03 & 769.59 & 1.57 \\
\hline & Jan/06 & 29.20 & 7.79 & 5.41 & 50.26 & 577.82 & 4.26 \\
\hline & Apr/06 & 21.30 & 7.10 & 8.70 & 19.35 & 110.01 & 3.30 \\
\hline & Jul/06 & 17.07 & 6.36 & 11.60 & 24.88 & 676.80 & 2.86 \\
\hline & Oct/06 & 26.00 & 6.59 & 8.70 & 47.43 & 675.90 & 2.80 \\
\hline & Jan/07 & 25.93 & 5.80 & 9.20 & 39.76 & 825.80 & 4.50 \\
\hline & Apr/07 & 24.76 & 6.00 & 6.30 & 27.97 & 420.00 & 5.84 \\
\hline & Jul/07 & 16.65 & 5.92 & 9.80 & 64.07 & 511.65 & 6.62 \\
\hline
\end{tabular}

Table 1. Water column mean values and standard deviation for temperature, $\mathrm{pH}$, dissolved oxygen, total phosphorus, total nitrogen and chlorophyll at the sampling stations of Chavantes Reservoir during the study period. * Stratified in summer. ** Only surface samples. 
The nutrient phosphorus and nitrogen exhibited a remarkable spatial and temporal variation in the accumulation reservoir. Higher concentrations were measured in the zones under influence of tributaries, as well as a decreasing tendency in direction to the dam. For the run-of-river reservoir the variation was not so regular.

Higher values of chlorophyll were generally observed in the lotic compartments of both reservoirs, especially in case of Salto Grande. In this reservoir values varied between 0,48 e $5,1 \mu \mathrm{g} \mathrm{L} \mathrm{L}^{-1}$. Seasonally higher concentrations were measured at PB, PRM, NB and SGD in February/2006, with the maximum in NB.

\begin{tabular}{|c|c|c|c|c|c|c|c|c|}
\hline & & $\begin{array}{c}\text { Cond. }\left(\mu \mathrm{S} \mathrm{cm}^{-1}\right) \\
\mathrm{M} \pm \mathrm{D} . \mathrm{P}\end{array}$ & $\begin{array}{c}\text { Turb. (NTU) } \\
\text { M } \pm \text { D.P }\end{array}$ & $\begin{array}{c}\text { T.S. }\left(\mathrm{mg} \mathrm{L}^{-1}\right) \\
M \pm D . P\end{array}$ & & $\begin{array}{c}\text { Cond. }\left(\mu \mathrm{S} \mathrm{cm}^{-1}\right) \\
\mathrm{M} \pm \mathrm{D} . \mathrm{P}\end{array}$ & $\begin{array}{c}\text { Turb. (NTU) } \\
\text { M } \pm \text { D.P }\end{array}$ & $\begin{array}{c}\text { T.S. }\left(\mathrm{mg} \mathrm{L}^{-1}\right) \\
\text { M } \pm \text { D.P }\end{array}$ \\
\hline \multirow{8}{*}{$\mathrm{UCH}$} & Oct/05 & $56.00 \pm 0.00$ & $4.74 \pm 0.27$ & $2.15 \pm 0.84$ & \multirow[t]{8}{*}{$\mathrm{CHD}^{*}$} & $60.00 \pm 3.74$ & $2.86 \pm 0.19$ & $1.13 \pm 0.15$ \\
\hline & Jan/06 & $44.00 \pm 1.41$ & $3.27 \pm 1.05$ & $2.13 \pm 0.91$ & & $47.75 \pm 5.56$ & $1.85 \pm 0.60$ & $0.85 \pm 0.41$ \\
\hline & Apr/06 & $80.00 \pm 0.00$ & $1.38 \pm 1.51$ & $1.63 \pm 0.23$ & & $90.18 \pm 1.34$ & $8.73 \pm 1.30$ & $1.38 \pm 0.22$ \\
\hline & Jul/06 & $60.67 \pm 2.58$ & $3.60 \pm 0.98$ & $3.53 \pm 1.66$ & & $67.32 \pm 4.47$ & $9.69 \pm 18.01$ & $1.02 \pm 0.73$ \\
\hline & Oct/06 & $80.00 \pm 0.00$ & $7.26 \pm 2.22$ & $2.47 \pm 1.05$ & & $90.00 \pm 0.00$ & $3.94 \pm 0.96$ & $1.11 \pm 0.29$ \\
\hline & Jan/07 & $100.00 \pm 0.00$ & $13.40 \pm 0.61$ & $5.82 \pm 4.25$ & & $104.63 \pm 5.03$ & $8.85 \pm 3.14$ & $2.51 \pm 0.56$ \\
\hline & Apr/07 & $64.06 \pm 0.25$ & $13.88 \pm 7.99$ & $2.37 \pm 0.60$ & & $63.77 \pm 2.50$ & $3.42 \pm 1.91$ & $1.88 \pm 1.33$ \\
\hline & Jul/07 & $62.00 \pm 0.00$ & $5.10 \pm 0.05$ & $3.63 \pm 1.01$ & & $84.18 \pm 0.87$ & $1.09 \pm 0.28$ & $0.60 \pm 0.02$ \\
\hline \multirow{8}{*}{$\mathrm{FA}^{*}$} & Oct/05 & $55.00 \pm 1.63$ & $7.65 \pm 1.04$ & $2.82 \pm 0.11$ & \multirow[t]{8}{*}{$\mathrm{VR}^{* *}$} & 42.00 & 36.30 & 27.80 \\
\hline & Jan/06 & $49.00 \pm 2.71$ & $8.69 \pm 8.87$ & $2.61 \pm 0.37$ & & 33.00 & 70.20 & 81.03 \\
\hline & Apr/06 & $90.91 \pm 2.92$ & $10.33 \pm 6.17$ & $2.30 \pm 0.30$ & & 70.00 & 42.00 & 28.74 \\
\hline & Jul/06 & $70.00 \pm 0.00$ & $1.47 \pm 0.78$ & $3.79 \pm 0.95$ & & 50.00 & 34.00 & 14.10 \\
\hline & Oct/06 & $90.00 \pm 0.00$ & $7.73 \pm 1.40$ & $2.30 \pm 0.15$ & & 80.00 & 173.00 & 103.35 \\
\hline & Jan/07 & $110.00 \pm 0.00$ & $11.95 \pm 0.62$ & $3.16 \pm 0.26$ & & 90.00 & 63.90 & 65.13 \\
\hline & Apr/07 & $69.73 \pm 2.78$ & $7.87 \pm 1.68$ & $1.28 \pm 0.72$ & & 47.00 & 810.00 & 302.43 \\
\hline & Jul/07 & $75.75 \pm 0.52$ & $4.24 \pm 1.77$ & $1.34 \pm 0.22$ & & 39.00 & 48.80 & 40.76 \\
\hline \multirow{8}{*}{$\mathrm{RC}^{*}$} & Oct $/ 05$ & $53.75 \pm 4.35$ & $5.21 \pm 0.85$ & $3.70 \pm 2.10$ & \multirow[t]{8}{*}{$\mathrm{IR}^{* *}$} & 67.00 & 69.20 & 107.30 \\
\hline & Jan/06 & $39.75 \pm 2.99$ & $8.06 \pm 2.65$ & $6.22 \pm 6.07$ & & 51.00 & 45.60 & 57.26 \\
\hline & Apr/06 & $93.24 \pm 4.75$ & $9.30 \pm 1.43$ & $1.34 \pm 0.05$ & & 120.00 & 19.00 & 10.94 \\
\hline & Jul/06 & $70.00 \pm 0.00$ & $1.09 \pm 0.36$ & $3.23 \pm 1.32$ & & 100.00 & 29.00 & 13.69 \\
\hline & Oct/06 & $90.00 \pm 0.00$ & $5.09 \pm 0.78$ & $1.53 \pm 0.16$ & & 120.00 & 44.00 & 45.17 \\
\hline & Jan/07 & $110.00 \pm 0.00$ & $9.28 \pm 1.77$ & $2.64 \pm 0.15$ & & 100.00 & 76.30 & 81.23 \\
\hline & Apr/07 & $70.47 \pm 4.25$ & $2.96 \pm 0.71$ & $1.75 \pm 0.39$ & & 85.00 & 45.70 & 45.45 \\
\hline & Jul/07 & $70.08 \pm 0.36$ & $2.54 \pm 0.70$ & $0.84 \pm 0.23$ & & 66.00 & 55.70 & 35.14 \\
\hline
\end{tabular}

Table 2. Water column mean values and standard deviation for conductivity, turbidity and suspended solids at the sampling stations of Chavantes Reservoir during the study period. * Stratified in summer. ${ }^{* *}$ Only surface samples.

The ANOVA test results evidenced the spatial and temporal differences for the limnological variables. For most variables the differences were significant when compared the sampling stations and the sampling periods for both reservoirs (Table 4).

A high percentage of data variance was explained by the PCA analysis (linear correlations), $88 \%$ for Chavantes and $81 \%$ for Salto Grande (Figure 7). The results evidence the riverreservoir spatial compartmentalization.

Transparency was associated to deeper sites in Chavantes (CHD and RC) - first component (positive side). Conversely, these variables (depth and transparency) were inversely correlated with suspended solids, turbidity, total phosphorus and total nitrogen, which were associated to the negative side of the first component and to the sampling stations VR and IR. 


\begin{tabular}{|c|c|c|c|c|c|c|c|c|c|c|}
\hline & & $\begin{array}{l}\text { Temp. } \\
\left({ }^{\circ} \mathrm{C}\right)\end{array}$ & $\mathrm{pH}$ & $\begin{array}{l}\text { O.D. } \\
\text { (mg L) }\end{array}$ & $\begin{array}{l}\text { Cond. } \\
\left(\mu \mathrm{S} \mathrm{cm}^{-1}\right)\end{array}$ & $\begin{array}{l}\text { Turb. } \\
\text { (NTU) }\end{array}$ & $\begin{array}{c}\text { T.S. } \\
\left(\mathrm{mg} \mathrm{L}^{-1}\right)\end{array}$ & $\begin{array}{c}\mathrm{TP} \\
\left(\mu \mathrm{g} \mathrm{L}^{-1}\right)\end{array}$ & $\begin{array}{c}\mathrm{TN} \\
\left(\mu \mathrm{g} \mathrm{L}^{-1}\right)\end{array}$ & $\begin{array}{l}\text { Chlor. } \\
\left(\mu \mathrm{g} \mathrm{L}^{-1}\right)\end{array}$ \\
\hline & & M \pm D.P. & $\mathrm{M} \pm \mathrm{D} . \mathrm{P}$ & $\mathrm{M} \pm \mathrm{D} . \mathrm{P}$ & $\mathrm{M} \pm \mathrm{D} . \mathrm{P}$ & $\mathrm{M} \pm \mathrm{D} . \mathrm{P}$ & $\mathrm{M} \pm \mathrm{D} . \mathrm{P}$ & $\mathrm{M} \pm$ D.P & $\mathrm{M} \pm \mathrm{D} . \mathrm{P}$ & $\mathrm{M} \pm$ D.P \\
\hline \multirow[t]{8}{*}{ USG } & Nov/05 & $24.60 \pm 0.00$ & $7.88 \pm 0.01$ & $7.66 \pm 0.02$ & $77.60 \pm 0.55$ & $5.99 \pm 0.23$ & $4.51 \pm 0.44$ & $27.08 \pm 0.81$ & $241.98 \pm 56.13$ & $1.99 \pm 0.97$ \\
\hline & Feb/06 & $25.70 \pm 0.00$ & $6.24 \pm 0.11$ & $7.98 \pm 0.04$ & $74.00 \pm 5.48$ & $7.67 \pm 3.79$ & $4.87 \pm 1.32$ & $15.25 \pm 2.10$ & $211.82 \pm 27.06$ & $1.52 \pm 0.08$ \\
\hline & May/06 & $21.10 \pm 0.00$ & $7.24 \pm 0.12$ & $9.20 \pm 0.00$ & $60.00 \pm 0.00$ & $0.33 \pm 0.58$ & $1.10 \pm 0.26$ & $14.19 \pm 0.00$ & $430.14 \pm 0.00$ & $1.07 \pm 0.00$ \\
\hline & Aug/06 & $20.37 \pm 0.00$ & $6.88 \pm 0.04$ & $9.24 \pm 0.05$ & $90.00 \pm 0.00$ & $2.22 \pm 0.72$ & $1.55 \pm 0.31$ & $9.87 \pm 1.41$ & $269.03 \pm 13.92$ & $0.79 \pm 0.15$ \\
\hline & Nov/06 & $26.25 \pm 0.01$ & $6.71 \pm 0.01$ & $10.05 \pm 0.05$ & $90.00 \pm 0.00$ & $2.50 \pm 0.45$ & $1.38 \pm 0.30$ & $6.36 \pm 0.43$ & $355.37 \pm 27.08$ & $2.09 \pm 0.41$ \\
\hline & Feb/07 & $27.19 \pm 0.01$ & $6.79 \pm 0.01$ & $8.08 \pm 0.08$ & $100.00 \pm 0.00$ & $4.65 \pm 0.00$ & $12.72 \pm 1.11$ & $17.73 \pm 3.06$ & $294.20 \pm 51.37$ & $2.40 \pm 0.12$ \\
\hline & May/07 & $22.30 \pm 0.00$ & $6.98 \pm 0.03$ & $9.58 \pm 0.04$ & $65.83 \pm 0.41$ & $2.91 \pm 0.39$ & $3.07 \pm 0.17$ & $11.74 \pm 1.39$ & $242.23 \pm 33.64$ & $1.40 \pm 0.17$ \\
\hline & Aug/07 & $19.62 \pm 0.04$ & $5.77 \pm 0.01$ & $9.40 \pm 0.00$ & $56.00 \pm 0.00$ & $3.09 \pm 0.34$ & $2.25 \pm 0.17$ & $35.20 \pm 45.86$ & $270.10 \pm 5.38$ & $1.21 \pm 0.37$ \\
\hline \multirow[t]{8}{*}{ PB } & Nov/05 & $24.54 \pm 0.05$ & $7.60 \pm 0.05$ & $7.58 \pm 0.11$ & $47.20 \pm 1.64$ & $5.50 \pm 0.62$ & $2.51 \pm 0.57$ & $28.26 \pm 1.46$ & $318.02 \pm 75.11$ & $1.12 \pm 0.16$ \\
\hline & Feb/06 & $26.80 \pm 0.00$ & $6.44 \pm 0.05$ & $8.00 \pm 0.00$ & $70.00 \pm 0.00$ & $4.60 \pm 0.89$ & $16.22 \pm 16.09$ & $17.77 \pm 2.75$ & $238.37 \pm 11.70$ & $2.21 \pm 1.27$ \\
\hline & May/06 & $21.40 \pm 0.00$ & $7.10 \pm 0.18$ & $9.10 \pm 0.00$ & $60.00 \pm 0.00$ & $1.40 \pm 0.89$ & $1.57 \pm 0.49$ & $14.88 \pm 0.31$ & $421.64 \pm 4.38$ & $1.24 \pm 0.20$ \\
\hline & Aug/06 & $20.69 \pm 0.01$ & $6.94 \pm 0.04$ & $6.36 \pm 0.23$ & $90.00 \pm 0.00$ & $6.00 \pm 0.00$ & $1.20 \pm 0.00$ & $12.68 \pm 0.23$ & $248.37 \pm 30.91$ & $0.78 \pm 0.44$ \\
\hline & Nov/06 & $25.83 \pm 0.05$ & $6.89 \pm 0.08$ & $8.40 \pm 0.00$ & $90.00 \pm 0.00$ & $3.25 \pm 0.85$ & $1.70 \pm 0.29$ & $7.72 \pm 1.48$ & $333.52 \pm 30.85$ & $1.59 \pm 0.18$ \\
\hline & Feb/07 & $26.16 \pm 0.02$ & $6.30 \pm 0.03$ & $7.55 \pm 0.05$ & $100.00 \pm 0.00$ & $42.43 \pm 3.09$ & $18.00 \pm 1$ & $25.08 \pm 1.62$ & $355.93 \pm 35.80$ & $2.02 \pm 0.30$ \\
\hline & May/07 & $23.10 \pm 0.00$ & $6.93 \pm 0.03$ & $9.38 \pm 0.04$ & $69.67 \pm 0.52$ & $1.60 \pm 0.16$ & $1.47 \pm 0.37$ & $9.82 \pm 0.20$ & $892.41 \pm 1122.30$ & $1.03 \pm 0.28$ \\
\hline & Aug/07 & $20.22 \pm 0.04$ & $5.83 \pm 0.02$ & $9.32 \pm 0.04$ & $61.17 \pm 0.41$ & $4.01 \pm 0.52$ & $2.04 \pm 0.73$ & $34.62 \pm 46.93$ & $279.07 \pm 9.58$ & $0.67 \pm 0.06$ \\
\hline \multirow[t]{8}{*}{ PRM } & Nov/05 & $25.65 \pm 0.06$ & $7.94 \pm 0.05$ & $7.48 \pm 0.35$ & $58.40 \pm 0.55$ & $12.47 \pm 1.85$ & $9.27 \pm 4.31$ & $65.03 \pm 13.58$ & $401.92 \pm 23.51$ & $3.82 \pm 0.16$ \\
\hline & Feb/06 & $24.70 \pm 0.00$ & $6.56 \pm 0.51$ & $8.34 \pm 0.09$ & $80.00 \pm 0.00$ & $356.67 \pm 5.77$ & $83.97 \pm 55.96$ & $40.83 \pm 4.22$ & $357.07 \pm 41.01$ & $5.10 \pm 2.71$ \\
\hline & May/06 & $17.95 \pm 0.05$ & $7.04 \pm 0.05$ & $9.80 \pm 0.00$ & $70.00 \pm 0.00$ & $26.33 \pm 3.51$ & $13.30 \pm 2.42$ & $34.68 \pm 1.71$ & $481.17 \pm 0.00$ & $1.69 \pm 0.23$ \\
\hline & Aug/06 & $21.13 \pm 0.01$ & $7.11 \pm 0.02$ & $8.50 \pm 0.35$ & $120.00 \pm 0.00$ & $12.39 \pm 0.00$ & $2.85 \pm 0.40$ & $48.04 \pm 2.36$ & $455.67 \pm 16.84$ & $0.83 \pm 0.06$ \\
\hline & Nov/06 & $27.30 \pm 0.33$ & $6.79 \pm 0.04$ & $7.16 \pm 0.09$ & $120.00 \pm 0.00$ & $12.80 \pm 0.80$ & & $31.19 \pm 0.56$ & $420.52 \pm 51.46$ & $1.07 \pm 0.10$ \\
\hline & Feb/07 & $24.33 \pm 0.02$ & $6.57 \pm 0.02$ & $8.20 \pm 0.07$ & $114.00 \pm 5.48$ & $44.37 \pm 6.68$ & $20.78 \pm 5.59$ & $28.18 \pm 3.06$ & $429.77 \pm 71.24$ & $1.54 \pm 0.03$ \\
\hline & May/07 & $20.80 \pm 0.00$ & $6.76 \pm 0.05$ & $9.50 \pm 0.00$ & $82.00 \pm 0.00$ & $9.56 \pm 2.37$ & $16.67 \pm 1.20$ & $37.48 \pm 2.28$ & $514.03 \pm 100.14$ & $1.59 \pm 0.42$ \\
\hline & Aug/07 & $19.08 \pm 0.13$ & $5.95 \pm 0.04$ & $9.44 \pm 0.21$ & $72.80 \pm 1.30$ & $18.07 \pm 4.13$ & $8.38 \pm 4.14$ & $25.18 \pm 1.73$ & $342.77 \pm 259.26$ & $0.78 \pm 0.44$ \\
\hline \multirow[t]{8}{*}{ NB } & Nov/05 & $24.80 \pm 0.00$ & $7.43 \pm 0.00$ & $7.54 \pm 0.00$ & $68.00 \pm 0.00$ & $15.00 \pm 0.00$ & $22.03 \pm 2.25$ & $52.84 \pm 0.00$ & $389.93 \pm 0.00$ & $4.16 \pm 1.43$ \\
\hline & Feb/06 & $24.70 \pm 0.00$ & $5.87 \pm 0.21$ & $7.67 \pm 0.57$ & $50.00 \pm 0.00$ & $130.00 \pm 0.00$ & $47.75 \pm 1.73$ & $34.29 \pm 0.00$ & $350.49 \pm 0.00$ & $4.36 \pm 0.60$ \\
\hline & May/06 & $17.96 \pm 0.04$ & $6.74 \pm 0.16$ & $10.27 \pm 0.15$ & $40.00 \pm 0.00$ & $23.67 \pm 4.04$ & $4.53 \pm 0.06$ & $29.27 \pm 0.00$ & $211.31 \pm 0.00$ & $1.35 \pm 0.00$ \\
\hline & Aug/06 & $20.80 \pm 0.00$ & $6.71 \pm 0.01$ & $7.57 \pm 0.06$ & $80.00 \pm 0.00$ & $11.50 \pm 0.00$ & $2.86 \pm 0.00$ & $28.29 \pm 0.86$ & $239.75 \pm 29.77$ & $1.01 \pm 0.16$ \\
\hline & Nov/06 & $26.15 \pm 0.38$ & $6.03 \pm 0.01$ & $7.40 \pm 0.00$ & $80.00 \pm 0.00$ & $11.50 \pm 0.71$ & $4.33 \pm 0.21$ & $12.94 \pm 1.58$ & $278.70 \pm 13.72$ & $3.60 \pm 0.32$ \\
\hline & Feb/07 & $23.55 \pm 0.01$ & $6.47 \pm 0.01$ & $8.25 \pm 0.07$ & $90.00 \pm 0.00$ & $58.00 \pm 36.77$ & $13.66 \pm 0.11$ & $15.83 \pm 0.81$ & $330.00 \pm 3.39$ & $1.01 \pm 0.32$ \\
\hline & May/07 & $20.84 \pm 0.00$ & $6.39 \pm 0.02$ & $9.45 \pm 0.07$ & $53.00 \pm 0.00$ & $6.86 \pm 0.00$ & $14.01 \pm 0.39$ & $17.35 \pm 2.64$ & $286.75 \pm 11.10$ & $3.93 \pm 1.11$ \\
\hline & Aug/07 & $18.74 \pm 0.02$ & $5.49 \pm 0.08$ & $9.30 \pm 0.14$ & $45.50 \pm 0.71$ & $10.30 \pm 0.14$ & $4.53 \pm 0.06$ & $10.63 \pm 1.17$ & $380.50 \pm 51.19$ & $1.91 \pm 0.16$ \\
\hline \multirow[t]{8}{*}{ SGD } & Nov/05 & $25.52 \pm 0.66$ & $7.83 \pm 0.05$ & $7.84 \pm 0.03$ & $49.60 \pm 0.55$ & $5.93 \pm 1.22$ & $2.88 \pm 1.23$ & $48.65 \pm 14.68$ & $295.59 \pm 155.43$ & $1.09 \pm 0.57$ \\
\hline & Feb/06 & $26.00 \pm 0.00$ & $6.28 \pm 0.04$ & $8.80 \pm 0.00$ & $70.00 \pm 0.00$ & $76.50 \pm 4.51$ & $22.86 \pm 2.41$ & $29.77 \pm 1.14$ & $303.71 \pm 32.26$ & $3.76 \pm 1.19$ \\
\hline & May/06 & $20.44 \pm 0.15$ & $6.84 \pm 0.15$ & $9.26 \pm 0.05$ & $60.00 \pm 0.00$ & $2.50 \pm 1.73$ & $1.10 \pm 0.26$ & $14.45 \pm 0.62$ & $304.61 \pm 23.59$ & $0.82 \pm 0.06$ \\
\hline & Aug/06 & $20.61 \pm 0.02$ & $6.92 \pm 0.03$ & $9.50 \pm 0.17$ & $90.00 \pm 0.00$ & $3.37 \pm 1.59$ & $1.63 \pm 0.10$ & $13.08 \pm 0.62$ & $297.87 \pm 10.46$ & $0.61 \pm 0.29$ \\
\hline & Nov/06 & $26.10 \pm 0.34$ & $6.79 \pm 0.10$ & $8.24 \pm 0.15$ & $97.50 \pm 4.63$ & $6.47 \pm 0.59$ & $1.66 \pm 0.23$ & $19.14 \pm 4.02$ & $456.18 \pm 17.95$ & $1.31 \pm 0.23$ \\
\hline & Feb/07 & $25.53 \pm 0.43$ & $6.57 \pm 0.02$ & $7.65 \pm 0.05$ & $110.00 \pm 0.00$ & $54.83 \pm 7.00$ & $14.76 \pm 2.31$ & $27.80 \pm 1.87$ & $396.57 \pm 96.56$ & $1.63 \pm 0.17$ \\
\hline & May/07 & $22.45 \pm 0.08$ & $6.76 \pm 0.01$ & $9.30 \pm 0.00$ & $72.00 \pm 0.93$ & $7.19 \pm 1.62$ & $4.89 \pm 0.63$ & $84.75 \pm 110.04$ & $315.03 \pm 15.20$ & $0.99 \pm 0.26$ \\
\hline & Aug/07 & $20.34 \pm 0.24$ & $5.95 \pm 0.06$ & $9.38 \pm 0.10$ & $62.13 \pm 1.25$ & $4.75 \pm 1.25$ & $1.38 \pm 0.16$ & $10.41 \pm 0.34$ & $318.13 \pm 9.41$ & $0.48 \pm 0.03$ \\
\hline
\end{tabular}

Table 3. Water column mean values and standard deviation for temperature, $\mathrm{pH}$, dissolved oxygen, conductivity, turbidity, suspended solids, total phosphorus, total nitrogen and chlorophyll at the sampling stations of Salto Grande Reservoir during the study period.

Total nitrogen and total phosphorus were also associated to periods of intense precipitation (second component).

In Salto Grande the variables suspended solids, turbidity, total phosphorus and chlorophyll were correlated (first component positive side) with the sampling sites PRM and NB 
(eventually SGD) during most sampling periods. Inversely, the variables transparency and depth were associated to PB and USG (eventually SGD).

\begin{tabular}{|c|c|c|c|c|c|}
\hline & & \multicolumn{2}{|c|}{ Chavantes } & \multicolumn{2}{|c|}{ Salto Grande } \\
\hline & & $\mathrm{F}$ & $\mathrm{p}$ & $\mathrm{F}$ & $\mathrm{p}$ \\
\hline \multirow{2}{*}{ Temperature } & Site & 0.79 & 0.56 & 3.92 & 0.01 \\
\hline & Month & 16.11 & 0.00 & 55.14 & 0.00 \\
\hline \multirow{2}{*}{$\mathrm{pH}$} & Site & 1.52 & 0.20 & 11.00 & 0.00 \\
\hline & Month & 19.95 & 0.00 & 68.42 & 0.00 \\
\hline \multirow{2}{*}{ Dissolved oxygen } & Site & 3.89 & 0.00 & 1.42 & 0.25 \\
\hline & Month & 4.08 & 0.00 & 6.97 & 0.00 \\
\hline \multirow{2}{*}{ Conductivity } & Site & 18.29 & 0.00 & 10.73 & 0.00 \\
\hline & Month & 45.11 & 0.00 & 25.70 & 0.00 \\
\hline \multirow{2}{*}{ Turbidity } & Site & 29.82 & 0.00 & 1.73 & 0.16 \\
\hline & Month & 1.96 & 0.08 & 2.85 & 0.02 \\
\hline \multirow{2}{*}{ Total N } & Site & 20.48 & 0.00 & 2.72 & 0.04 \\
\hline & Month & 42.38 & 0.00 & 1.25 & 0.30 \\
\hline \multirow{2}{*}{ Total P } & Site & 15.10 & 0.00 & 3.89 & 0.01 \\
\hline & Month & 8.41 & 0.00 & 1.48 & 0.21 \\
\hline \multirow{2}{*}{ Suspended solids } & Site & 57.44 & 0.00 & 3.49 & 0.01 \\
\hline & Month & 1.70 & 0.13 & 5.50 & 0.00 \\
\hline \multirow{2}{*}{ Chlorophyll } & Site & 9.03 & 0.00 & 4.17 & 0.00 \\
\hline & Month & 2.74 & 0.02 & 5.20 & 0.00 \\
\hline \multirow{2}{*}{ Transparency } & Site & 120.13 & 0.00 & 17.42 & 0.00 \\
\hline & Month & 10.84 & 0.00 & 7.32 & 0.00 \\
\hline
\end{tabular}

Table 4. Results of the ANOVA two-way (F and $\mathrm{p}$ ) for the limnological variables tested for sampling points and sampling periods. Significant differences $(p<0.05)$ in bold. For spatial comparison freedom degree is 5 for Chavantes and 4 for Salto Grande. For temporal comparison freedom degree is 7 for both reservoirs.
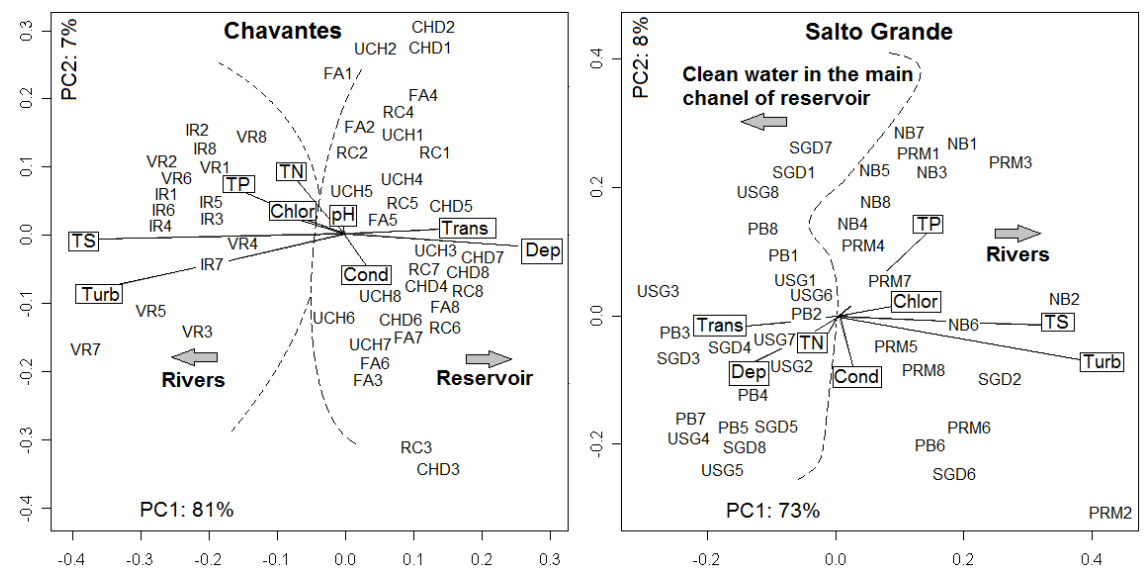

Fig. 7. Graphic results of the PCA analysis (components 1 and 2) for ordination of the limnological variables considering the sampling sites and sampling periods for Chavantes and Salto Grande Reservoirs. 


\section{Discussion}

The rainfall pattern was similar for both reservoirs, with higher values in summer (December-February) and lower in autumn/winter (April to September). This seasonal regime had already been identified as an important factor influencing the limnological functioning of the Paranapanema River reservoirs (Nogueira et al., 2002b, 2006; Jorcin \& Nogueira, 2005; Pagioro et al., 2005 b; Henry et al., 2006).

Higher values of turbidity, suspended solids, nutrients and conductivity were observed in the tributary rivers mouths for both reservoirs. However, only a minor influence of these rivers entrance was observed in the main water body of the accumulation reservoir, Chavantes, due to its large size and high water retention time. Conversely, for the run-ofriver reservoir the influence of the tributaries was prominent, with major changes of the water quality downstream the rivers entrance, towards to the dam zone. This process is particularly evident in the rainy period (summer).

Our results corroborate previous studies carried out in Chavantes and Salto Grande by Pagioro et al. (2005 a) and Nogueira et al. (2006). These authors considered Chavantes as an oligotrophic system, eventually mesotrophic in some periods of the year, and Salto Grande a meso/eutrophic system, due to the introduction of large nutrient loads from the lateral tributaries.

In Chavantes, different from Salto Grande, the longitudinal zones (sensu Thornton et al., 1990) were clearly observed. This spatial organization is a consequence of the high water retention time, large size and complex morphometry, which determine the occurrence of distinct compartments.

The water retention time is key variable for reservoir ecology, interfering on physical, chemical and biological characteristics and it depends on the interactions among distinct factors, such as precipitation, outflow, evaporation an infiltration (Tundisi, 1990; Nogueira et al., 1999; Nogueira 2000; Nogueira et al., 2006). The vertical structure of the water column in Chavantes and Salto Grande is directly associated to the water retention time features of each reservoir.

Except for the winter season, the deeper sampling points located in Chavantes Reservoir (CHD, RC and FA) exhibited a well-defined thermal and chemical stratification. The temperature profiles mean values varied up to $11^{\circ} \mathrm{C}$ (from 18 to $29^{\circ} \mathrm{C}$ ). The oxygen and $\mathrm{pH}$ were much lower in deep layer, indicating reduced chemical conditions. Thomaz et al. (1997) also found stratification only in the dam (lacustrine) zone of Segredo Reservoir in the Iguaçu River (Paraná State, Brazil).

Pagioro et al. (2005b) and Nogueira et al. (2006) had already observed stratification in the water column of larger reservoirs of the Paranapanema basin during spring and summer and complete mixture in winter. These authors pointed out to the occurrence of low oxygen concentrations in the bottom layers of Chavantes. Henry \& Nogueira (1999) also verified seasonal thermocline in Jurumirim Reservoir, the first in the Paranapanema reservoir cascade. Other similar patterns in reservoirs of southeast and central Brazil have been registered. De Filippo et al. (1999) observed low oxygen concentrations, and even anoxic condition, bellow $20 \mathrm{~m}$ depth in Serra da Mesa Reservoir (Goiás State, Brazil), right after its filling up and still under strong influence of decomposition of organic loads. Clinograde 
patterns were measured by Pinto-Coelho et al. (2006) in São Simão Reservoir, located in Parnaíba River (border of Goiás and Minas Gerais states, Brazil).

Continuous vertical mixing and absence of thermal and chemical stratification in Salto Grande are associated to the reservoir semi-lotic conditions (very low water retention time) and low depth. This condition was also observed by Pagioro et al. (2005b) in run-of-river reservoirs of Paraná State.

When compared the maximum temperature of both reservoirs it is observed low values in Salto Grande. The low heat retention capacity in this reservoir is also associated to its morphometry and functioning (run-of-river system). As a small size reservoir, Salto Grande is also more influenced by the tributary entrances, which exhibit colder waters in some periods of the year, especially in the winter.

The contrast in terms of retention time and morphometry among Chavantes and Salto Grande also explain the distinct magnitude of the tributaries influence. The modifications due to the tributaries entrance in the run-of-river is higher, especially bellow the Pardo River Mouth (PRM). There is no sufficient time/distance for sedimentation of the introduced loads. The simple morphometry do not favor the depositional process as well. The opposite situation occurs in Chavantes, due to its dendritic shape and long main axis, which favor the particles settlement and a longitudinal increase in transparency.

In the sampling station CHD, the deepest one in Chavantes, it was observed high transparency and low values of turbidity and suspended solids. This characteristic corroborates the predictable modifications towards the dam zone due to the reduction of water velocity and increase in depth (Thornton et al., 1990; Henry \& Maricatto, 1996; Thomaz et al., 1997; Nogueira et al., 1999; Pinto-Coelho et al., 2006).

The sampling points located in the transitional (river-reservoir) zone of Chavantes, exhibited some common characteristics, such as higher values of turbidity and total nitrogen and lower transparency, when compared to the lacustrine zone. However, in relation to dissolved oxygen there was contrasting tendencies. Higher concentrations were observed at $\mathrm{UCH}$, probably due to the predominant high water velocity and relatively low depth. Conversely, at FA the values were low, associated to the water column stratification and reduced concentrations in the bottom. Besides the input of sediments from agriculture activities, in this region there is also the influence of erosive process of the river banks associated to intensive wind action. The sediments are deposited in deep layers, bellow the mixture zone (ca. 20m), where is verified an increase in turbidity.

In Salto Grande the sampling points located in the reservoir main axis exhibited similarity for some limnological variables. Nevertheless, the effect of the Pardo River entrance in the intermediate region of the reservoir is remarkable, with the increase of nutrients, turbidity and diminution of transparency. It is clearly observed the distinctiveness of the water masses, the brownish one coming from Pardo River and the greenish and much more transparent one from the Paranapanema River (lacustrine zone of Chavantes). Sampaio et al. (2002), Britto (2003) and Nogueira et al. (2006) have already mentioned the effects of Pardo River on the increase of turbidity in Salto Grande.

Comparisons between different kinds of reservoirs show the occurrence of higher concentrations of turbidity and suspended solids in run-of-river systems, which are more similar to rivers in terms of hydrodynamics (Pagioro et al., 2005 b; Nogueira et al., 2006). 
The Novo River (NB), despite its reduced flow, is another important tributary contributing to the introduction of nutrients and suspended solids (and decrease of transparency) for the dam zone of Salto Grande (SGD). In NB there are extensive macrophytes banks and also episodic algal blooms, indicate by chlorophyll peaks, due to high nutrient concentration and reduced flow.

The transportation and retention of suspended particles, from river inputs, are considered as the main processes determining the reservoirs compartmentalization in terms of their limnology features and organization of biotic communities (Thornton et al., 1990).

The sampled lotic stretches exhibited mean values for the limnolgical variables distinct from the ones calculated for the reservoirs (more lacustrine) sampling sites, as evidenced by ANOVA tests.

The discrimination among the riverine sampling points, VR, IR, USG e PB; the tributaries mouth zones, PRM e NB; the river-reservoir transitional compartments, UCH, FA e SGD and the lentic environments, RC e CHD, was possible through the obtained results. For the riverine compartments there was two different conditions; rivers without upstream dams (Verde, Itararé, Pardo and Novo) and stretches under dams influence (USG and PB). In the first case there is a direct response of the watershed to precipitation events and the predominance of lower temperature, especially in autumn and winter season, higher dissolved oxygen, nutrient concentration, turbidity and suspended solids and lower transparency. Some of these characteristics are amplified by the intensive land use for agriculture (Brigante et al., 2003; Nogueira et al., 2006; Pinto-Coelho et al., 2006).

Feitosa et al. (2006) verified that the environmental degradation is more intensive in the lateral tributaries compared to the Paranapanema River main channel and reservoirs. In our study, except for the oxygen values, this tendency was also verified.

A strong influence of Chavantes Reservoir on the upstream sampling points of Salto Grande Reservoir (upstream the Pardo River entrance) was evident for some periods. The storage reservoir releases transparent waters with low concentrations of suspended solids, because most sediments are retained in upstream compartments due to its large size, complex morphometry and high water retention time, as previously mentioned. Similar structure and functioning was already described for Jurumirim Reservoir, the first in the cascade and about $50 \mathrm{~km}$ upstream the transitional zone of Chavantes (Henry \& Maricatto, 1996; Nogueira et al., 1999).

In January/2007 it was observed high conductivity values in Chavantes Reservoir, including the sampling points without the influence of the tributaries, such as RC and CHD. This fact can be related to three causes. The first would be a consequence of the superficial water intake to the turbines, promoting a water mass displacement, including the ones coming from the tributaries, towards the dam zone. The second cause would be related to the sudden water level increase, which inundates exposed littoral areas during the dry season where the vegetation grew and its decomposition would change the physical and chemical characteristics of water. Finally, the high frequency and volumes of rains in this period would promote a significant runoff of superficial adjacent soils, resulting in the introduction of suspended and dissolved material.

In relation to the conductivity it is also important to note the relatively low values of the Verde River (VR), especially if compared to the ones measured in the Itarare River (IR). 
Despite their proximity, the considered rivers must have particular geological substrate responsible for the observed differences. Another hypothesis is that the land use (urban and agriculture activities) in Itararé basin would be more intensive. In fact in this basin it is observed intensive commercial forestation with Pinus sp. and Eucaliptus sp., for instance.

Despite of the relative higher loads of nutrients in Salto Grande, associated to intensive human activities in the tributaries watersheds and around the reservoir, the chlorophyll concentrations in this reservoir was low. Certainly this fact is associated to the very low water retention time. Probably an increase in the WRT would result in higher phytoplankton density and biomass, as commonly observed in other tropical reservoirs exposed to human induced eutrophication. Some examples are provided by the literature such as in Billings and Guarapiranga Reservoirs (Sendacz \& Kubo, 1999) and Garças Lake (reservoir) (Sant'Anna et al., 1997; Bicudo et al., 1999) in São Paulo metropolitan region; Paranoá Reservoir in Brasília metropolitan region (Branco \& Cavalcanti, 1999) and Iraí Reservoir in Curitiba metropolitan region (Bollmann et al., 2005; Pagioro et al., 2005a). According to Abe et al. (2006), in Brazil the highest concentration of nutrients potentially important for eutrophication (nitrogen and phosphorus) are mainly associated to urban development.

\section{Conclusion}

In order to represent the main results of this study it is presented a schematic drawing (Figure 8 ) of the studied reservoirs, synthesizing the main limnological differences. The storage reservoir showed intense thermal and chemical stratifications in summer and isothermal and isochemical profiles in winter, what is directed associated to the physical characteristics and operational functioning. The run-of-river reservoir exhibited only daily (ephemerons) thermal stratification tendency near the dam during summer, what was also a consequence of its physical characteristics and operational procedures.

The influence of the accumulation reservoir on the run-of-river reservoir, downstream located, seems to be less intense in summer and more evident in winter (dry period).

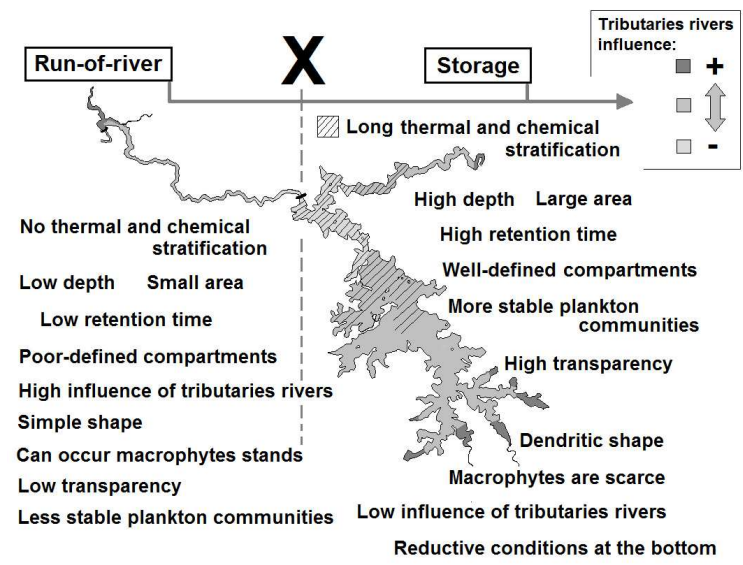

Fig. 8. Schematic drawing synthesizing the main limnological differences between Chavantes (storage) and Salto Grande (run-of-river) Reservoirs. 
The distinctiveness between both reservoirs, despite their geographical proximity, was verified. Nevertheless, the second hypothesis was not confirmed as the influence of the lacustrine zone of upstream reservoir was limited to certain periods of the year. The tributaries had a strong influence on the run-of-river reservoir, modifying the turbidity and nutrient concentrations after their mouths, especially in the rainy season.

\section{Acknowledgements}

The authors are grateful to Fapesp for financial support (process 2005-02811-0) and for the scholarship conceived for the second author (process 2005/03311-0); to Silvia M. C. Casanova and Fabiana Akemi Kudo for collaboration during the field work and laboratory analyses; to Duke Energy - Geração Paranapanema for financial and logistic support and for the hydrological data and to Casa de Agricultura de Fartura (SP) and Instituto de Águas do Paraná (PR) for the precipitation data.

\section{References}

Abe, D.S.; Tundisi, J.G.; Matsumura-Tundisi, T.; Tundisi, J.E.M.; Sidagis Galli, C.; TeixeiraSilva, V.; Afonso, G.F.; Von Haehling, P.H.A.; Moss, G. \& Moss, M. (2006). Monitoramento da qualidade ecológica das águas interiores superficiais e do potencial trófico em escala continental no Brasil com uso de hidroavião, In: Eutrofização na América do Sul: causas, conseqüências e tecnologia de gerenciamento e controle. São Carlos: Instituto Internacional de Ecologia, J.G. Tundisi, T. MatsumuraTundisi, \& C.S. Galli. (Eds.), 225-239, DMD Propaganda, São Carlos, Brazil

Agostinho, A.A.; Gomes, L.C. \& Pelicice, F.M. (2007). Ecologia e manejo de recursos pesqueiros em reservatórios do Brasil. Eduem, Maringá, Brazil

Bicudo, C.E.M.; Ramírez, R.J.J.; Tucci, A. \& Bicudo, D.C. (1999). Dinâmica de populações fitoplanctônicas em ambiente eutrofizado: o lago das Garças, São Paulo, In: Ecologia de Reservatórios: estrutura, função e aspectos sociais, R. Henry, (Ed.), 451-507, Fapesp/Fundibio, Botucatu, Brazil

Bollmann, H.A. \& Andreoli, O.R. (2005). Água no sistema urbano, In: Gestão Integrada de Manaciais de Abastecimento Eutrofizados, C.V. Andreoli \& C. Carneiro, (Eds.), 83-119, Capital, Curitiba, Brazil

Branco, C.W.C. \& Cavalcanti, C.G.B. (1999). A ecologia das comunidades planctônicas no lago Paranoá, In: Ecologia de Reservatórios: Estrutura, Função e Aspectos Sociais, R. Henry, (Ed.), 575-595, Fapesp/Fundibio, Botucatu, Brazil

Brigante, J.; Espíndola, E.L.G.; Povinelli, J. \& Nogueira, A.M. (2003). Caracterização física, química e biológica da água do rio Mogi-Guaçu, In: Limnologia fluvial, J. Brigante, \& E.L.G. Espíndola, (Eds.), 55-76, Rima, São Carlos, Brazil

Britto, Y.C.T. (2003). Associações de Cladocera (Crustacea Branchiopoda) do sistema de reservatórios em cascata do rio Paranapanema (SP-PR). Dissertação de mestrado, UNESP, Botucatu, Brazil

Cole, G.A. (1979) Textbook of limnology (2nd.), The C.V. Mosby Company, Saint Louis

De Fellipo, R.; Gomes, E.L.; Lenz-César, J.; Soares, C.B.P. \& Menezes, C.F.S. (1999). As alterações na qualidade de água durante o enchimento do reservatório da UHE Serra da Mesa (GO), In: Ecologia de reservatórios: estrutura, função e aspectos sociais, R. Henry, (Ed.), 324-345, Fapesp/Fundibio, Botucatu, Brazil 
Feitosa, M.F.; Nogueira, M.G. \& Vianna, N.C. Transporte de Nutrientes e Sedimentos no Rio Paranapanema (SP/PR) e seus Principais Tributários nas Estações Seca e Chuvosa. In: Ecologia de reservatórios: Impactos potenciais, ações de manejo e sistemas em cascata, M.G. Nogueira, R. Henry \& A. Jorcin, (Eds.), 435-459, Rima, São Carlos, Brazil.

Han, B.; Armengol, J.; Garcia, J. C.; Comerna, M R.; Dolz. J. \& Straskraba, M. (2000). The thermal structure of Sal Reservoir (NE: Spain): a simulation approach. Ecological Modelling, Vol.125, pp. 109-122

Henry, R. \& Gouveia, L. (1993). O fluxo de nutrientes e seston em cursos de água do alto Paranapanema (São Paulo) - sua relação com usos do solo e morfologia das bacias de drenagem. Na. Acad. Bras. Ciênc, Vol.65, pp. 439-51

Henry, R.; Carvalho, E.D.; Nogueira, M.G.; Pompeo, M.L.M.; Moschini-carlos, V.; Santos, C.M. dos; Luciano, S. de C. \& Fujihara C.Y. (1995), The Jurumirim Reservoir, In: Mid. Congress Excursions, XXVI SIL Congress, R. Henry \& P.A.C. Senna (Eds.), 13-33, São Paulo, Brazil

Henry, R. \& Maricatto, F.E. (1996). Sedimentation rates of tripton in Jurumirim Reservoir (São Paulo, Brasil). Limnologica, Vol.25, pp. 15-25.

Henry, R. \& Nogueira, M.G. (1999). A Represa de Jurumirim (São Paulo): Primeira síntese sobre o conhecimento limnológico e uma proposta preliminar de manejo ambiental, In: Ecologia de reservatórios: estrutura, função e aspectos sociais, R. Henry, (Ed.), 651685, Fapesp/Fundibio, Botucatu, Brazil

Henry, R. (1992). The oxygen deficit in Jurumirim Reservoir (Paranapanema River, São Paulo, Brazil). Jpn. J. Limnol, Vol.53, pp. 379-84

Henry, R.; Nogueira, M.G.; Pompeo, M.L.L. \& Moschini-Carlos, V. (2006). Annual and shortterm variability in primary productivity by phytoplankton and correlated abiotic factors in the Jurumirim Reservoir (São Paulo, Brazil). Braz. J. Biol., Vol.66, pp. 239261

Jorcin, A. \& Nogueira, M.G. (2005) Temporal and spatial patterns along the cascade of reservoirs in the Paranapanema River (SE Brazil) based on the characteristics of sediment and sediment-water interface. Lakes $\mathcal{E}$ Reserv. Res. Manage., Vol.10, pp. 112

Kelman, J.; Pereira, M.V.; Araripe-Neto, T.A. \& Sales P.R.H. (1999). Hidroeletrecidade, In: Águas doces no Brasil: Capital ecológico, uso e conservação, A.C. Rebouças, B. Braga \& Tundisi J.G, (Eds.), 371-418, Editora Escrituras, São Paulo, Brazil

Kindt, R. \& Coe, R. (2005). Tree diversity analysis. World Agroforestry Centre, Kenya

Marckereth, F.I.H.; Heron J. \& Talling J.F. (1978) Water analysis: some revised methods for limnologists. Freshwater Biological Association, London

Mercante, C.T.J. \& Tucci-Moura, A. (1999). Comparação entre os índices de Carlson e de Carlson modificado aplicados a dois ambientes aquáticos subtropicais, São Paulo, SP. Acta Limnologica Brasiliensia, Vol.11, No.1, pp. 1-14

Naliato, D.A.O.; Nogueira, M.G. \& Perbiche-Neves, G. (2009). Discharge pulses of hydroelectric dams and their effects in the downstream limnological conditions: a case study in a large tropical river (SE Brazil). Lakes \& Reser.: Res. and Manag., Vol.14, pp. 301-314

Nogueira M.G.; Henry, R. \& Maricatto, F.E. (1999). Spatial and temporal heterogeneity in the Jurumirim Reservoir, São Paulo, Brazil. Lakes \& Reser.: Res. and Manag., Vol.4, pp. $107-120$ 
Nogueira, M.G.; Jorcin A.; Vianna, N.C. \& Britto Y.C. (2006). Reservatórios em cascata e os efeitos na limnologia e organização das comunidades bióticas (fitoplâncton, zooplâncton e zoobentos): Um estudo de caso no rio Paranapanema (SP/PR). In: Ecologia de reservatórios: Impactos potenciais, ações de manejo e sistemas em cascata, M.G. Nogueira, R. Henry \& A. Jorcin, (Eds.), 83-125, Rima, São Carlos, Brazil

Nogueira, M.G.; Reis-Oliveira, P.C. \& Britto Y.T. (2008) Zooplankton assemblages (Copepoda and Cladocera) in a cascade of reservoirs of a large tropical river (SE Brazil). Limnetica, Vol.27, pp. 151-170

Nogueira M.G. (2000). Phytoplankton composition, dominance and abundance as indicators of enviromental compartmentalization in Jurumirim Reservoir (Paranapanema River), São Paulo, Brazil. Hydrobiol., Vol.431, pp. 115-128

Nogueira, M.G.; Jorcin, A.; Vianna, N.C. \& Britto, Y.C.T. (2002a). Uma avaliação dos processos de eutrofização nos reservatórios em cascata do Rio Paranapanema (SPPR), Brasil, In: El Água en Iberoamerica, de la limnologia a a gestión en Sudamerica, Argentina, A. Cirelli \& G. Marquisa (Eds.), 91-106, Cyted XVII, Argentina

Nogueira, M.G.; Jorcin, A.; Vianna, N.C. \& Britto, Y.C.T. (2002b). A two- year study on the limnology of a cascade reservoir system in a large tropical river in Southeast Brazil. 4th International conference on reservoir limnology and water quality, Èeské Bud jovice, August, 2002

Pagioro, T.A.; Thomaz, S.M. \& Roberto, M.C. (2005a). Caracterização limnológica abiótica dos reservatórios. In: Biocenoses em reservatórios: padrões espaciais e temporais, L. Rodrigues, S.M. Thomaz, A.A. Agostinho \& L.C. Gomes (Eds.), 17-37, Rima, São Carlos, Brazil

Pagioro, T.A.; Velho, L.F.M.; Lansac-Tôha, F.A.; Pereira, D.G. \& Nakamura, A.K.S. (2005b). Influência do grau de trofia sobre os padrões de abundância de bactérias e protozoários planctônicos em reservatórios do Estado do Paraná. In: Biocenoses em reservatórios: padrões espaciais e temporais, L. Rodrigues, S.M. Thomaz, A.A. Agostinho \& L.C. Gomes (Eds.), 47-56, Rima, São Carlos, Brazil

Perbiche-Neves, G. \& Nogueira, M.G. (2010). Multidimensional effects on cladoceran (Crustacea, Anomopoda) assemblages in two cascade reservoirs (SE - Brazil). Lakes and Res.: Research and Manag., Vol.15, pp. 151-164

Perbiche-Neves, G.; Ferreira, R.A.R. \& Nogueira, M.G. (2011). Phytoplankton structure in two contrasting cascade reservoirs (Paranapanema River, Southeast Brazil). Biologia (Bratislava) Section Botany, Vol. 66 (6), pp. 967-976

Pinto-Coelho, R.M.; Azevedo, L.M.; Rizzi, P.E.V.; Bezerra-Neto, J.F. \& Rolla, M.E. (2006). Origens e efeitos do aporte externo de nutrientes em um reservatório tropical de grande porte: Reservatório de São Simão (MG/GO), In: Ecologia de reservatórios: Impactos potenciais, ações de manejo e sistemas em cascata, M.G. Nogueira, R. Henry \& A. Jorcin, (Eds), 127-164, Rima, São Carlos, Brazil

R Development Core Team (2009). A language and environment for statistical computing. Vienna, Austria, R Foundation for Statistical Computing ISBN 3-900051-07-0, Date access 15.04.2009, available from: <http:/ / www.R-project.org>.

Sampaio, E.V.; Rocha, O.; Matsumura-Tundisi, T. \& Tundisi, J.G. (2002). Composition and Abundance of Zooplankton in the Limnetic of Seven Reservoir of the Paranapanema River, Brazil. Braz. J. Biol., Vol.62, No.3, pp. 525-545

Sant'Anna, C.L.; Sormus, L.; Tucci, A. \& Azevedo, M.T.P. (1997). Variação sazonal do fitoplâncton do lago da Garças, São Paulo, SP, Brasil. Hoehnea, Vol.24. pp. 67-86 
Sendacz, S. \& Kubo, E. (1999). Zooplâncton de reservatórios do alto Tietê, Estado de São Paulo, In: Ecologia de reservatórios: estrutura, função e aspectos sociais, R. Henry, (Ed.), 511-529, Fapesp/Fundibio, Botucatu, Brazil

Soares, M.C.S.; Marinho, M.M.; Huszar, V.L.M.; Branco, C.W.C. \& Azevedo, S.M.F.O. (2008) The effects of water retention time and watershed features on the limnology of two tropical reservoirs in Brazil. Lakes \& Reserv.: Res. and Manag., Vol.13, pp. 257-269

StatSoft, Inc. (2006) STATISTICA (data analysis software system), version 6.0 Available from: $<$ www.statsoft.com>

Stevaux, J.C.; Martins, D.P. \& Meurer, M. (2009). Changes in regulated tropical rivers: The Paraná River downstream Porto Primavera dam, Brazil. Geomorphology, Vol.113, pp. 230-238

Strickland, J.D. \& Parsons, T.R. (1960). A manual of sea water analysis. Bull. Fish. Res. Board of Can., Vol.125, pp. 1-185

Talling, J.F. \& Driver D. (1963). Some problems in the estimation of chlorophyll a in phytoplankton. In: Proceedings, Conference of primary productivity measurements in marine and freshwater, 142-146, Atomic Energy Commision, Hawaii, USA

Thomaz, S.M.; Bini, L.M. \& Alberti, S.M. (1997). Limnologia do reservatório de Segredo: padrões de variação espacial e temporal, In: Reservatório de Segredo: bases ecológicas para o manejo, A.A. Agostinho \& L.C. Gomes, (Eds.), 19-37, Eduem, Maringá, Brazil

Thornton, W.K. (1990). Perspectives on reservoir limnology, In: Reservoir Limnology: ecological perspectives, K.W. Thornton, B.L. Kimmel \& E.F. Payne (Eds.), 1-13, John Wiley \& Sons Inc., New York

Tundisi, J.G. (1990). Key factors of reservoir functioning and geographical aspects of reservoir limnology - chairman's overview. Arch. Hydrobiol. Beih. Ergebn., Vol.33, pp. 654-646

Tundisi, J.G. \& Matsumura-Tundisi, T. (2003) Integration of research and management in optimizing multiple uses of reservoirs: the experience in South America and Brazilian case studies. Hydrobiologia, Vol.500, pp. 231-42

Valderrama, J.G. (1981). The simultaneous analysis of total nitrogen and phosphorus in natural waters. Mar. Chem., Vol.10, pp. 109-122 


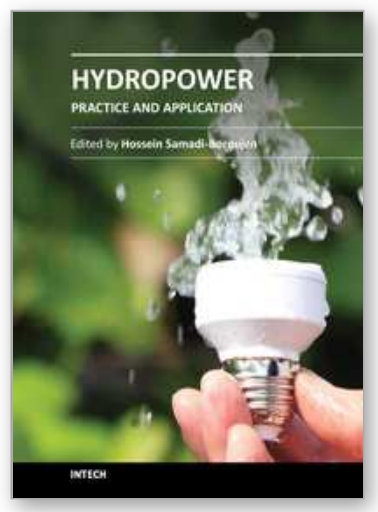

\author{
Hydropower - Practice and Application \\ Edited by Dr. Hossein Samadi-Boroujeni
}

ISBN 978-953-51-0164-2

Hard cover, 320 pages

Publisher InTech

Published online 09, March, 2012

Published in print edition March, 2012

Hydroelectric energy is the most widely used form of renewable energy, accounting for 16 percent of global electricity consumption. This book is primarily based on theoretical and applied results obtained by the authors during a long time of practice devoted to problems in the design and operation of a significant number of hydroelectric power plants in different countries. It was preferred to edit this book with the intention that it may partly serve as a supplementary textbook for students on hydropower plants. The subjects being mentioned comprise all the main components of a hydro power plant, from the upstream end, with the basin for water intake, to the downstream end of the water flow outlet.

\title{
How to reference
}

In order to correctly reference this scholarly work, feel free to copy and paste the following:

Marcos Gomes Nogueira, Gilmar Perbiche-Neves and Danilo A. O. Naliato (2012). Limnology of Two Contrasting Hydroelectric Reservoirs (Storage and Run-of-River) in Southeast Brazil, Hydropower - Practice and Application, Dr. Hossein Samadi-Boroujeni (Ed.), ISBN: 978-953-51-0164-2, InTech, Available from: http://www.intechopen.com/books/hydropower-practice-and-application/limnology-of-two-contrastinghydroelectric-reservoirs-storage-and-run-of-river-in-southeast-brazil

\section{INTECH}

open science | open minds

\section{InTech Europe}

University Campus STeP Ri

Slavka Krautzeka 83/A

51000 Rijeka, Croatia

Phone: +385 (51) 770447

Fax: +385 (51) 686166

www.intechopen.com

\section{InTech China}

Unit 405, Office Block, Hotel Equatorial Shanghai

No.65, Yan An Road (West), Shanghai, 200040, China

中国上海市延安西路65号上海国际贵都大饭店办公楼 405 单元

Phone: +86-21-62489820

Fax: +86-21-62489821 
(C) 2012 The Author(s). Licensee IntechOpen. This is an open access article distributed under the terms of the Creative Commons Attribution 3.0 License, which permits unrestricted use, distribution, and reproduction in any medium, provided the original work is properly cited. 